\title{
Effectiveness of predicting in-hospital mortality in critically ill children by assessing blood lactate levels at admission
}

Zhenjiang Bai ${ }^{1 \dagger}$, Xueping Zhu ${ }^{2 \dagger}$, Mengxia $\mathrm{Li}^{3}$, Jun Hua ${ }^{1}$, Ying $\mathrm{Li}^{1}$, Jian Pan ${ }^{4}$, Jian Wang ${ }^{4}$ and Yanhong $\mathrm{Li}^{3,4^{*}}$

\begin{abstract}
Background: Hyperlactatemia upon admission is a documented risk factor for mortality in critically ill adult patients. However, the predictive significance of a single lactate measurement at admission for mortality in the general population of critically ill children remains uncertain. This study evaluated the predictive value of blood lactate levels at admission and determined the cut-off values for predicting in-hospital mortality in the critically ill pediatric population.
\end{abstract}

Methods: We enrolled 1109 critically ill children who were admitted to a pediatric intensive care unit between July 2008 and December 2010. Arterial blood samples were collected in the first 2 hours after admission, and the lactate levels were determined. The Pediatric Risk of Mortality III (PRISM III) scores were calculated during the first 24 hours after admission.

Results: Of the 1109 children admitted, 115 (10.4\%) died in the hospital. The median (interquartile range) blood lactate level in critically ill children was $3.2 \mathrm{mmol} / \mathrm{l}$ (2.2-4.8). Among the children, 859 (77.5\%) had a lactate concentration $>2.0 \mathrm{mmol} / \mathrm{l}$. The blood lactate level upon admission was significantly associated with mortality (odds ratio $[O R]=1.38 ; 95 \%$ confidence interval $[\mathrm{Cl}], 1.30-1.46 ; p<0.001$ ), even after adjustment for age, gender, and illness severity assessed by PRISM III (OR $=1.27 ; p<0.001)$. Multivariate regression analysis showed that a high blood lactate level $(\mathrm{OR}=1.17 ; 95 \% \mathrm{Cl}, 1.07-1.29 ; p=0.001)$, a high PRISM III score $(\mathrm{OR}=1.15 ; 95 \% \mathrm{Cl}, 1.11-1.20 ; p<0.001)$, and a low serum albumin $(\mathrm{OR}=0.92 ; 95 \% \mathrm{Cl}, 0.88-0.96 ; p<0.001)$ were independent risk factors for mortality in critically ill children. Blood lactate achieved an area under-the-receiver-operating-characteristic curve (AUC) of 0.79 ( $p<0.001$ ) for predicting mortality that was similar to that of PRISM III (AUC $=0.82 ; p<0.001$ ). The $p$-value for a comparison of both AUCS was 0.318 . Blood lactate displayed a sensitivity of $61 \%$ and a specificity of $86 \%$ in predicting mortality at the optimal cut-off value of $5.55 \mathrm{mmol} / \mathrm{l}$, and the positive and negative likelihood ratios were 4.5 and 0.45 , respectively.

Conclusions: A high blood lactate level at admission is independently associated with and predictive of in-hospital mortality in the general population of critically ill children.

Keywords: Blood lactate, Critically ill children, Cut-off value, In-hospital mortality, Pediatric risk of mortality III (PRISM III), Predictive test

\footnotetext{
* Correspondence: lyh072006@hotmail.com

${ }^{\dagger}$ Equal contributors

${ }^{3}$ Department of Nephrology, Children's Hospital affiliated to Soochow

University, Suzhou, China

${ }^{4}$ Institute of Pediatric Research, Children's Hospital affiliated to Soochow

University, Suzhou, China

Full list of author information is available at the end of the article
} 


\section{Background}

Early recognition of children who are at high risk for mortality allows for timely changes in therapy and improves the overall outcomes. Although critical care in pediatric patients has changed dramatically over the last several decades, there has been no consistent marker for obtaining predictions of mortality in a general population of critically ill children.

Lactate has been used as a marker of tissue hypoperfusion and cellular hypoxia, and hyperlactatemia is significantly associated with mortality [1-5]. Previous studies evaluated the predictive value for mortality of both a single lactate screening measurement at admission and serial lactate measurements $[1,6]$. Hyperlactatemia in adult patients upon admission to a general medical intensive care unit was a predictive marker identifying patients who were at high risk for death [7].

Despite the publication of a few studies that investigated the association of admission hyperlactatemia with mortality in the pediatric population [6,8-16], information on its importance in a general population of critically ill children is limited $[9,14,15]$. The predictive value of a single lactate level assessed at admission, unlike serial measurements of lactate, is controversial in the general population of critically ill children $[9,14,15]$. One study of 75 patients admitted to the pediatric intensive care unit (PICU) found that blood lactate levels assessed at 24 hours after admission, but not at 6 hours, had better sensitivity or specificity as a predictor of death [15]. Persistent hyperlactatemia 24 hours after PICU admission is associated with mortality, as shown in a study of 50 patients with early hyperlactatemia (lactate $>2 \mathrm{mmol} / \mathrm{l}$ within 6 hours of admission). In that study group, however, the lactate level measured at admission did not differ significantly between survivors and non-survivors [9]. In contrast to these reports, a recently published clinical study conducted in a retrospective cohort suggested that the blood lactate concentration at PICU admission predicts mortality independent of the pediatric index of mortality (PIM) [14].

The predictive significance for a single lactate measurement as a screening method for children upon admission to the PICU remains uncertain. Furthermore, a lactate level that consistently predicts mortality in the general population of critically ill children has not been identified. We hypothesized that the blood lactate level upon admission to PICU is significantly associated with mortality in critically ill children. This study evaluated the predictive value of admission blood lactate and determined the cut-off values for predicting in-hospital mortality in the general population of critically ill children.

\section{Methods}

All children admitted to the PICU from July 2008 to December 2010 were considered for inclusion in the study. The criteria for PICU admission were adopted from guidelines for developing admission and discharge policies for the pediatric intensive care unit [17]. The study exclusion criteria were death in the first 2 hours after admission and unexpectedly discharged or transferred to another hospital. The Institutional Review Board of the Children's Hospital of Soochow University approved the study. Informed consent was obtained from the parents of the children enrolled in the study.

\section{Clinical and laboratory data collection}

Clinical and laboratory data were collected on the day of admission and included age, gender, admission diagnosis, illness severity assessed by the Pediatric Risk of Mortality III (PRISM III) score, routine hematological tests, a serum biochemical profile, and arterial blood gas analysis. Clinical status, comorbidities, therapeutic interventions, and medication were recorded daily until hospital discharge or death.

The presence of multi-organ dysfunction syndrome (MODS) during hospitalization was determined according to the criteria modified from the international pediatric sepsis consensus conference [18]. The maximum number of dysfunctional organs was 6 (cardiovascular, respiratory, neurologic, hematologic, renal, and hepatic). Children with dysfunction in 3 or more organs were compared to those with fewer than 3 dysfunctional organs.

\section{The PRISM III score}

The PRISM III score was calculated to assess the illness severity according to methods described in the original studies $[19,20]$. In brief, PRISM III was scored based on agerelated physiological parameters collected during the first 24 hours after admission, including systolic blood pressure, heart rate, temperature, pupillary reflexes, mental status, acidosis ( $\mathrm{pH}$ and total $\mathrm{CO} 2$ ), $\mathrm{pCO} 2, \mathrm{pO} 2$, glucose, potassium, creatinine, blood urea, white blood cell count, platelet count, and prothrombin or partial thromboplastin time.

\section{Clinical outcome}

In-hospital mortality was defined as a death occurring in the hospital after PICU admission. A favorable outcome was defined as a child who was discharged to home directly from the PICU or after transfer to another department in our hospital.

\section{Blood sample collection and measurement}

To determine the blood lactate level at admission, an arterial sample was collected in the first 2 hours after a child's admission to the PICU. The sample was collected directly into a tube containing anticoagulant. The blood concentration of lactate was measured immediately using an automatic biomedical blood gas analyzer (CCX, NOVA Biomedical, Waltham, MA, USA) as part of a 
routine panel of blood gas tests in our clinical laboratory with the electrode method, and it was expressed in millimole per liter $(\mathrm{mmol} / \mathrm{l})$. The blood gas analyzer was calibrated every 2 hours and checked twice per day. The detection limit for lactate was $0.5 \mathrm{mmol} / \mathrm{l}$. The coefficient of variation was $7.5 \%$ at the low level and $5.0 \%$ at the high level. The normal range in children is 0.5$2.5 \mathrm{mmol} / \mathrm{l}$. The laboratory investigators were blinded to the sample sources and clinical outcomes.

\section{Statistical analysis}

Statistical analyses were done by using SPSS 13.0. Assumptions of normality and homogeneity of variance were first checked. For continuous variables with a skewed distribution, descriptive results were expressed as medians and interquartile ranges. The Mann-Whitney $U$ test was used to determine the differences between two groups, and the Kruskal-Wallis $\mathrm{H}$ test was used to analyze the differences among groups. Univariate binary and multivariate logistic regression analyses were performed to investigate whether blood lactate was independently associated with in-hospital mortality. The model fit was assessed with the Hosmer-Lemeshow goodness-of-fit test. A non-significant value for the Hosmer-Lemeshow Chi-square test suggests an absence of biased fit. Analysis of the area under the curve (AUC) of the Receiver Operating Characteristic (ROC) curve was constructed to assess the predictive strength. The nonparametric method of Delong was used to compare significant difference between AUCs (Sigmaplot 10.0 software). Sensitivity, specificity, and positive and negative likelihood ratios and predictive values were calculated at different cut-off values. Optimal cut-off points to maximize both sensitivity and specificity were also determined. All probability values are two-sided. Differences with $p$ values $<0.05$ were considered to be statistically significant.

\section{Results}

\section{Patient characteristics}

The study enrolled 1109 critically ill children, including 1045 children with a medical admission diagnosis and 64 with a surgical admission diagnosis. Of the total 1204 children admitted to the PICU during the study period, 54 were first excluded: 37 because of refusal to participate by parents and 17 due to failure to obtain an arterial blood sample during the first 2 hours after admission. Of the 1150 children who had lactate values from arterial blood samples, 41 were excluded: 2 died in the first 2 hours after admission, 3 were transferred to another hospital, and 36 were unexpectedly discharged due to economic reasons. Major medical admission diagnoses included respiratory diseases (39.9\%), neurological diseases (24.3\%), gastrointestinal diseases (7.3\%), cardiovascular diseases (5.1\%), sepsis (4.0\%), hematologic/oncologic diseases (3.7\%), poisoning (2.9\%), and others (7.0\%). There was no significant difference between the included and excluded children with regard to age (median [interquartile range]: 1.00 [0.25-3.00] vs 0.67 [0.25-3.00] years, $p=0.221$ ), gender (male/female: $689 / 420$ vs $23 / 18, p=0.513)$, or PRISM III score (3 [2-5] vs 3 [0-7], $p=0.740)$.

Of the total 1109 children, 115 (10.4\%) died in the hospital. The median time from PICU admission to death was 48 hours (min-max range: [2.5-1176]; interquartile range [24-84]) after admission. Children who were discharged to home were considered to have a favorable outcome. The demographic and clinical characteristics and laboratory findings on the day of admission are compared between survivors and non-survivors in Table 1. The concentration of blood lactate at admission in surviving children was significantly higher than in those who did not survive ( $\mathrm{p}<0.001)$.

\section{Comparison of data in children with different concentrations of blood lactate}

Blood lactate was detectable with a range of 0.6$28.3 \mathrm{mmol} / \mathrm{l}$ in 1101 samples. For undetectable levels,

\begin{tabular}{|c|c|c|c|}
\hline Characteristics & $\begin{array}{l}\text { Survivors } \\
(n=994)\end{array}$ & $\begin{array}{c}\text { Non-survivors } \\
(n=115)\end{array}$ & $P$ value \\
\hline Age, years & $1.00[0.29-3.00]$ & $1.00[0.25-3.13]$ & 0.680 \\
\hline Gender, male/female & $629 / 365$ & $60 / 55$ & 0.025 \\
\hline PRISM III score & $3[0-6]$ & $12[5-23]$ & $<0.001$ \\
\hline MODS $\geq 3^{a}, n$ & $63(6.3)$ & $58(50.4)$ & $<0.001$ \\
\hline $\begin{array}{l}\text { Need for mechanical } \\
\text { ventilation }{ }^{b}, n\end{array}$ & $172(17.3)$ & $67(58.3)$ & $<0.001$ \\
\hline \multicolumn{4}{|c|}{ Laboratory findings on admission day ${ }^{c}$} \\
\hline Blood lactate, $\mathrm{mmol} / \mathrm{l}$ & $3.10[2.10-4.50]$ & $6.60[3.8-11.95]$ & $<0.001$ \\
\hline Serum albumin, g/l & $42.6[39.6-45.8]$ & $36.5[30.2-42.0]$ & $<0.001$ \\
\hline Blood glucose, mmol/l & $7.12[5.71-9.75]$ & $10.10[5.73-21.29]$ & $<0.001$ \\
\hline $\begin{array}{l}\text { Serum creatinine, } \\
\mu \mathrm{mol} / \mathrm{l}\end{array}$ & $27.9[23.20-36.78]$ & $42.7[27.45-67.65]$ & $<0.001$ \\
\hline $\begin{array}{l}\text { Blood urea nitrogen, } \\
\mu \mathrm{mol} / \mathrm{l}\end{array}$ & $3.71[2.61-5.08]$ & $4.90[3.69-6.71]$ & $<0.001$ \\
\hline $\begin{array}{l}\text { Serum total bilirubin, } \\
\mu \mathrm{mol} / \mathrm{l}\end{array}$ & $6.48[4.26-9.94]$ & $8.03[4.20-13.96]$ & 0.023 \\
\hline $\begin{array}{l}\text { Blood bicarbonate, } \\
\mathrm{mmol} / \mathrm{l}\end{array}$ & 20.8 [18.0-23.9] & $17.3[12.0-21.1]$ & $<0.001$ \\
\hline Arterial pH & $7.415[7.349-7.472]$ & $7.359[7.181-7.438]$ & $<0.001$ \\
\hline
\end{tabular}

Values are median [interquartile range]. Numbers in parentheses denote percentages.

MODS, multi-organ dysfunction syndrome; PRISM III, pediatric risk of mortality III. a MODS developed during hospitalization. ${ }^{b} \mathrm{Administration}$ during

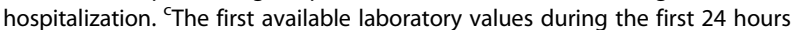
after admission. 
the values of blood lactate were arbitrarily given values of 0.4. The median blood lactate level measured in the first 2 hours after admission in critically ill children was $3.2 \mathrm{mmol} / \mathrm{l}$. Among the children, 859 (77.5\%) had a lactate concentration $>2.0 \mathrm{mmol} / \mathrm{l}$, including 485 (43.7\%), 204 (18.4\%), 79 (7.1\%), $34(3.1 \%)$ and 57 (5.1\%) who had lactate concentrations of 2.1-4.0, 4.16.0, 6.1-8.0, 8.1-10.0 and greater than $10.0 \mathrm{mmol} / \mathrm{l}$, respectively.

A comparison of the demographic and clinical characteristics and the laboratory findings collected on the day of admission among children is shown in Table 2 . The incidence of mortality in critically ill children was significantly associated with increased blood lactate levels $(p<0.001)$. A significant increase in the PRISM III scores $(p<0.001)$ and the blood glucose concentrations $(\mathrm{p}<0.001)$ was associated with increases in the blood lactate levels. In contrast, the blood bicarbonate concentrations $(\mathrm{p}<0.001)$ and arterial $\mathrm{pH}$ values $(\mathrm{p}<0.001)$ were significantly decreased with increases in the blood lactate levels.

\section{Association of blood lactate level with in-hospital mortality}

Univariate binary and multivariate logistic regression analyses were performed to investigate whether the blood lactate level at admission was independently associated with in-hospital mortality (Table 3). Age, gender, illness severity as assessed by the PRISM III score, and laboratory findings collected on the day of admission that were potentially associated with in-hospital mortality were included in the analyses. The following factors were significantly associated with in-hospital mortality in the unadjusted binary logistic regression analysis: gender, PRISM III score, blood lactate level, albumin, glucose, creatinine, urea nitrogen and bicarbonate, and arterial $\mathrm{pH}$ value. The odds for in-hospital mortality increased by $38 \%$, for every $1 \mathrm{mmol} / \mathrm{l}$ increase in blood lactate $(\mathrm{OR}=1.38 ; 95 \% \mathrm{CI}, 1.30-1.46 ; p<0.001)$. The association of blood lactate levels with in-hospital mortality remained significant after adjusting for age, gender, and the severity of illness as assessed by the PRISM III score $(\mathrm{OR}=1.27$; 95\% CI, 1.19-1.35; $p<0.001)$.

Table 2 Comparison of demographic and clinical characteristics and laboratory findings on admission day among children with different concentrations of blood lactate

\begin{tabular}{|c|c|c|c|c|c|c|c|}
\hline Admission blood lactate, $\mathrm{mmom} / \mathrm{l}$ & $0.0-2.0$ & $2.1-4.0$ & 4.1-6.0 & $6.1-8.0$ & $8.1-10.0$ & $>10.0$ & $P$ value \\
\hline$n$ & 250 & 485 & 204 & 79 & 34 & 57 & \\
\hline Age, years & $1.75[0.58-4.00]$ & $1.00[0.33-3.00]$ & $0.67[0.25-2.00]$ & $0.54[0.25-3.50]$ & $0.42[0.17-1.25]$ & $0.92[0.33-3.00]$ & $<0.001$ \\
\hline Gender, male/female & $163 / 87$ & $304 / 181$ & $122 / 82$ & $60 / 19$ & $9 / 25$ & $31 / 26$ & $<0.001$ \\
\hline PRISM III score & $3[0-6]$ & $3[0-6]$ & $3[0.75-7]$ & $5[2-11]$ & $8[4-15.75]$ & $14[6-24]$ & $<0.001$ \\
\hline $\operatorname{MODS} \geq 3^{a}, \mathrm{n}$ & $11(4.4)$ & $24(4.9)$ & $20(9.8)$ & $20(25.3)$ & $12(35.3)$ & $34(59.6)$ & $<0.001$ \\
\hline Mechanical ventilation ${ }^{\mathrm{b}}, \mathrm{n}$ & $29(11.6)$ & 95 (19.6) & $48(23.5)$ & $23(29.1)$ & $13(38.2)$ & $31(54.4)$ & $<0.001$ \\
\hline In-hospital mortality, n & $7(2.8)$ & $27(5.6)$ & $18(8.8)$ & $14(17.7)$ & $9(26.5)$ & $40(70.2)$ & $<0.001$ \\
\hline \multicolumn{8}{|l|}{ Laboratory findings on admission day ${ }^{c}$} \\
\hline \multirow{2}{*}{ Albumin, $g / l$} & 42.6 & 42.7 & 42.0 & 40.2 & 39.6 & 32.8 & \multirow{2}{*}{$<0.001$} \\
\hline & {$[39.1-45.8]$} & {$[39.8-46.0]$} & [39.9-45.7] & {$[34.2-44.5]$} & {$[35.9-43.15]$} & {$[29.0-40.0]$} & \\
\hline \multirow{2}{*}{ Glucose, mmol/l } & 6.50 & 6.90 & 7.60 & 8.90 & 11.35 & 16.27 & \multirow{2}{*}{$<0.001$} \\
\hline & {$[5.35-8.00]$} & {$[5.70-9.00]$} & [6.15-11.37] & {$[6.65-14.02]$} & {$[7.40-21.21]$} & {$[10.78-27.90]$} & \\
\hline \multirow{2}{*}{ Creatinine, $\mu \mathrm{mol} / /$} & 28.2 & 27.4 & 27.8 & 31.7 & 37.6 & 62.4 & \multirow{2}{*}{$<0.001$} \\
\hline & {$[23.2-35.5]$} & {$[23.2-35.0]$} & {$[23.1-37.5]$} & {$[22.8-48.8]$} & {$[25.9-60.1]$} & {$[43.0-79.7]$} & \\
\hline \multirow{2}{*}{ BUN, $\mu \mathrm{mol} / \mathrm{l}$} & 3.64 & 3.70 & 3.59 & 4.46 & 4.87 & 4.72 & \multirow{2}{*}{$<0.001$} \\
\hline & {$[2.62-5.04]$} & {$[2.62-4.92]$} & {$[2.35-5.12]$} & {$[2.85-6.69]$} & {$[3.04-6.48]$} & [3.80-6.92] & \\
\hline \multirow{2}{*}{ Total bilirubin, $\mu \mathrm{mol} / /$} & 6.08 & 6.50 & 7.01 & 9.43 & 4.91 & 6.71 & \multirow{2}{*}{$<0.001$} \\
\hline & {$[4.20-8.50]$} & {$[4.21-9.73]$} & [4.73-11.69] & [6.18-17.28] & {$[3.33-8.89]$} & {$[4.03-12.61]$} & \\
\hline \multirow{2}{*}{ Bicarbonate, $\mu \mathrm{mol} / /$} & 21.4 & 20.8 & 20.8 & 17.7 & 15.9 & 11.5 & \multirow{2}{*}{$<0.001$} \\
\hline & [18.8-24.4] & {$[18.4-24.0]$} & {$[17.9-23.8]$} & [14.8-21.4] & {$[11.7-20.4]$} & {$[6.6-15.3]$} & \\
\hline \multirow{2}{*}{ Arterial pH } & 7.439 & 7.421 & 7.390 & 7.368 & 7.267 & 7.177 & \multirow{2}{*}{$<0.001$} \\
\hline & [7.385-7.488] & [7.364-7.476] & [7.332-7.456] & [7.304-7.464] & [7.141-7.338] & {$[7.005-7.272]$} & \\
\hline
\end{tabular}

Values are median [interquartile range]. Numbers in parentheses denote percentages.

$B U N$, blood urea nitrogen; MODS, multi-organ dysfunction syndrome; PRISM III, pediatric risk of mortality III.

${ }^{a}$ MODS developed during hospitalization. ${ }^{b}$ Administration during hospitalization. ${ }^{\mathrm{c}}$ The first available laboratory values during the first 24 hours after admission. 
Table 3 Univariate and multivariate logistic regression analyses of variables potentially associated with inhospital mortality

\begin{tabular}{lcccccc}
\hline & \multicolumn{2}{c}{$\begin{array}{c}\text { Univariate binary logistic } \\
\text { regression }\end{array}$} & & \multicolumn{2}{c}{$\begin{array}{c}\text { Multivariate logistic } \\
\text { regression }\end{array}$} \\
\cline { 2 - 3 } \cline { 6 - 7 } & OR (95\% Cl) & $P$ value & & OR (95\% Cl) & $P$ value \\
\hline Age & $1.02(0.96-1.08)$ & 0.542 & & $1.06(0.96-1.16)$ & 0.249 \\
Gender & $1.58(1.07-2.33)$ & 0.021 & & $1.11(0.63-1.97)$ & 0.715 \\
PRISM III score & $1.18(1.15-1.21)$ & $<0.001$ & & $1.15(1.11-1.20)$ & $<0.001^{\mathrm{e}}$ \\
Laboratory findings on admission day & & & & \\
Lactate & $1.38(1.30-1.46)$ & $<0.001^{\mathrm{a}}$ & & $1.17(1.07-1.29)$ & $0.001^{\mathrm{b}, \mathrm{c}}$ \\
Albumin & $0.86(0.83-0.89)$ & $<0.001$ & & $0.92(0.88-0.96)$ & $<0.001^{\mathrm{d}}$ \\
Glucose & $1.11(1.08-1.13)$ & $<0.001$ & & $1.00(0.96-1.03)$ & 0.829 \\
Creatinine & $1.01(1.00-1.01)$ & $<0.001$ & & $0.99(0.98-1.01)$ & 0.428 \\
Urea nitrogen & $1.05(1.01-1.08)$ & 0.006 & & $1.03(0.94-1.12)$ & 0.556 \\
Total bilirubin & $1.01(1.00-1.01)$ & 0.010 & & $1.01(0.99-1.01)$ & 0.080 \\
Bicarbonate & $0.89(0.87-0.93)$ & $<0.001$ & & $1.03(0.98-1.08)$ & 0.217 \\
Arterial pH & $0.01(0.00-0.03)$ & $<0.001$ & & $0.54(0.06-4.75)$ & 0.580 \\
\hline
\end{tabular}

$\mathrm{Cl}$, confidence interval; $O R$, odds ratio; PRISM III, pediatric risk of mortality III. The $p$ value of the Hosmer-Lemeshow goodness-of-fit test for the multivariate logistic regression model was 0.611 .

${ }^{\text {aT }}$ The association of blood lactate with in-hospital mortality remained significant after adjustment for age, gender, and the PRISM III score (OR $=1.27 ; 95 \% \mathrm{Cl}$, 1.19-1.35; $p<0.001$ ). ${ }^{\mathrm{b}}$ The association of the blood lactate level with in-hospital mortality remained significant after adjustment for serum albumin and the PRISM score $(\mathrm{OR}=1.17 ; 95 \% \mathrm{Cl}, 1.09-1.26 ; p<0.001)$. ${ }^{\mathrm{C} O d d s}$ ratio represents the increase in risk per $1 \mathrm{mmom} / \mathrm{l}$ increase in lactate concentration. ${ }^{d}$ Odds ratio per $1 \mathrm{~g} / \mathrm{l}$ increase in albumin level. ${ }^{e}$ Odds ratio per 1-point increase in PRISM III score.

${ }^{\text {T}}$ The first available laboratory values during the first 24 hours after admission.

Multivariate logistic regression analysis identified blood lactate $(\mathrm{OR}=1.17 ; 95 \% \mathrm{CI}, 1.07-1.29 ; p=0.001)$, serum albumin (OR per $1 \mathrm{~g} / 1$ increase $=0.92$; $95 \% \mathrm{CI}, 0.88$ $0.96 ; p<0.001$ ), and the PRISM III score (OR per 1-point increase $=1.15$; $95 \% \mathrm{CI}, 1.11-1.20 ; p<0.001)$ as independent factors that were significantly associated with inhospital mortality in critically ill children. The HosmerLemeshow goodness-of-fit test for the multivariate logistic regression model was not significant $(p=0.611)$, indicating that the model adequately fits the data. Furthermore, the association between blood lactate levels and inhospital mortality remained significant after adjusting for the PRISM III score and serum albumin $(\mathrm{OR}=1.17$; 95\% CI, 1.09-1.26; $p<0.001$ ).

\section{Ability of blood lactate level to predict in-hospital mortality}

The predictive ability of blood lactate levels from all children admitted to the PICU $(\mathrm{n}=1109)$ for in-hospital mortality $(\mathrm{n}=115)$ was assessed (Table 4$)$. The level of blood lactate was predictive of in-hospital mortality and achieved AUC of 0.79 (95\% CI, 0.74-0.84; $p<0.001$ ). This AUC is similar to the PRISM III score (AUC $=0.82$; $95 \%$ CI, 0.78-0.86; $p<0.001$ ) for predicting the in-hospital mortality. The $p$-value for comparison of both AUCs was 0.318. Combining blood lactate levels with the PRISM III score improved the predictive performance (AUC $=0.86$; 95\% CI 0.83-0.90; $\mathrm{p}<0.001$ ), which is better than blood lactate alone $(\mathrm{p}=0.018)$, but not significantly better than PRISM alone $(\mathrm{p}=0.135)$. Figure 1 shows the ROC curves and the AUC of the blood lactate level at admission, the PRISM III score, and the combination of the blood lactate with the PRISM III score for predicting the in-hospital mortality of critically ill children.

The sensitivity and specificity of the blood lactate levels and PRISM III scores at the optimal cut-off value to predict in-hospital mortality are shown in Table 4 . Blood lactate displayed a sensitivity of $61 \%$ and a specificity of $86 \%$ at the optimal cut-off value of $5.55 \mathrm{mmol} / \mathrm{l}$. The positive and negative likelihood ratios were 4.5 and 0.45 , respectively. The PRISM III score displayed a sensitivity of $68 \%$ and a specificity of $82 \%$ for predicting inhospital mortality at the optimal cut-off score of 7.5, and the positive and negative likelihood ratios were 3.8 and 0.39 , respectively.

We also calculated the sensitivity and specificity of differing concentrations of blood lactate to predict inhospital mortality in critically ill children (Table 5). At the cut-off value of $>2.0 \mathrm{mmol} / \mathrm{l}$, blood lactate displayed a sensitivity of $94 \%$ and a specificity of $24 \%$ for predicting inhospital mortality, and the positive and negative likelihood ratios were 1.2 and 0.25 , respectively. The specificity increased to $98 \%$, and the positive likelihood ratio increased

Table 4 Predicting performance of admission blood lactate and PRISM III for in-hospital mortality

\begin{tabular}{|c|c|c|c|c|c|c|}
\hline & AUC & $95 \% \mathrm{Cl}$ & $P$ value & Optimal cut-off value & Sensitivity & Specificity \\
\hline Admission blood lactate & 0.79 & $0.74-0.84$ & $<0.001$ & $5.55 \mathrm{mmol} / \mathrm{l}$ & $61 \%$ & $86 \%$ \\
\hline PRISM III score & 0.82 & $0.78-0.86$ & $<0.001$ & 7.5 & $68 \%$ & $82 \%$ \\
\hline Blood lactate combined with PRISM III & 0.86 & $0.83-0.90$ & $<0.001$ & & $63 \%$ & $93 \%$ \\
\hline \multicolumn{7}{|c|}{$P$ value (comparison of the difference between AUCs) } \\
\hline \multicolumn{7}{|c|}{$p=0.318$ (between blood lactate and PRISM III) } \\
\hline \multicolumn{7}{|c|}{$p=0.135$ (between blood lactate combined with PRISM III and PRISM III alone) } \\
\hline \multicolumn{7}{|c|}{$p=0.018$ (between blood lactate combined with PRISM III and blood lactate alone) } \\
\hline
\end{tabular}

$A U C$, area under the receiver-operating-characteristic curve; $C l$, confidence interval; PRISM III, pediatric risk of mortality III. 


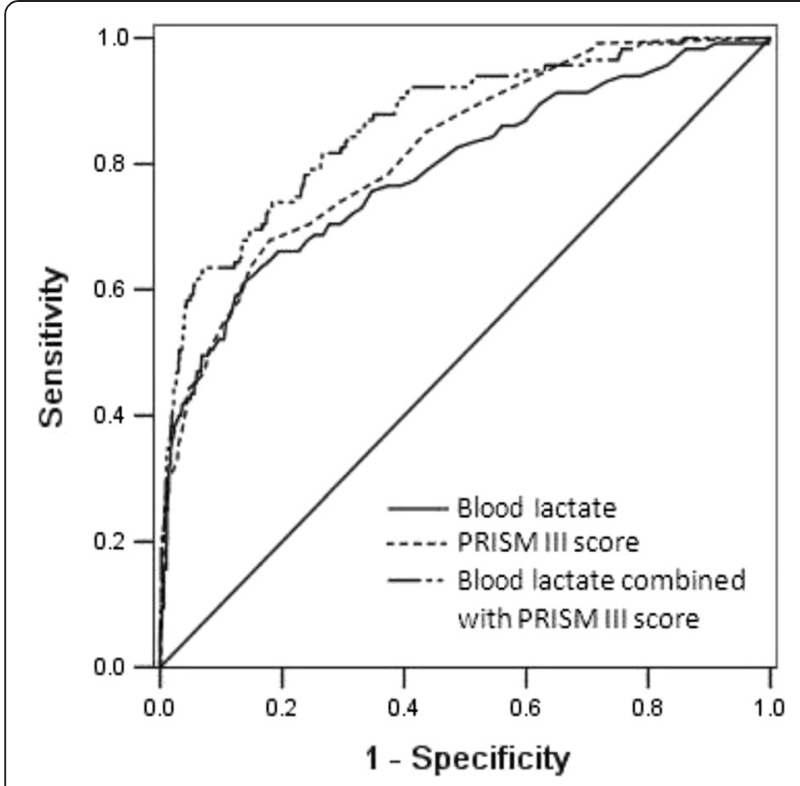

Figure 1 Receiver operating characteristic curves for the ability of blood lactate, PRISM III, and blood lactate combined with PRISM III to predict in-hospital mortality in generally critically ill children $(\mathbf{n}=\mathbf{1 1 0 9})$. The area under the receiver operating characteristic curve for blood lactate, PRISM III, and blood lactate combined with PRISM III were $0.79,0.82$ and 0.86 , respectively, with a Hosmer-Lemeshow goodness-of-fit $p$ value $>0.05$.

to 19.8 at the cut-off value of $>10.0 \mathrm{mmol} / \mathrm{l}$, although the sensitivity decreased to $34 \%$. The OR values of blood lactate at the levels above the set cut-off points are shown in Table 5 .

In addition, $65.0 \%$ children had a lactate concentration $>2.5 \mathrm{mmol} / \mathrm{l}$ in the study, which is suggested to be an optimal cut-off value for lactate to predict deterioration and mortality in adult patients [1]. Blood lactate displayed a sensitivity of $90 \%$ and a specificity of $38 \%$ to predict in-hospital mortality at this cut-off value in critically ill children.

\section{Discussion}

This study provides data on blood lactate concentrations in critically ill children and demonstrates that the blood lactate level upon admission to a general medical PICU is significantly associated with in-hospital mortality. A high blood lactate level at PICU admission is predictive of in-hospital mortality in critically ill children.

Many studies have demonstrated that either admission lactate or peak lactate concentration is associated with mortality in adults $[1,4,21,22]$. To our knowledge, a limited number of studies verified the use of hyperlactatemia as a prognostic index in critically ill children who are admitted to the PICU $[9,14,15]$. Our results are in line with recently published findings that suggest that blood lactate concentration upon admission to PICU is predictive of mortality, independent of PIM2. Patients with contemporaneous blood lactate and PIM2 measurements at PICU admission were enrolled in a retrospective cohort study [14]. Our study was conducted in a large mixed cohort of critically ill children. Blood samples were prospectively collected for assessing the blood lactate concentration. The observation that the extent of absolute hyperlactatemia is strongly linked with mortality independent of illness severity indicates that blood lactate is a useful early predictor in identifying critically ill children who are at high risk of death in the pediatric intensive care setting.

The discrepancy between our data and the data from two studies performed on a small number of patients might most likely be attributed to the small sample size in the previous studies $[9,15]$. However, the possibility that the discrepancy might be caused by differences in the course of the clinical illness at PICU admission must be considered. Our study included a higher proportion of patients (859 of $1109,77 \%$ ) with a high level of blood lactate at admission ( $>2 \mathrm{mmol} / \mathrm{l}$ ), compared to a previous publication in which high values were found in only 50 of the 705 studied children at admission (7\%) [9]. Together with the high overall mortality of $10.6 \%$ in the present study, this finding implies that children might be admitted later to our PICU, most likely because of delayed PICU transfers.

One contribution of this study is the use of PRISM III to control for the severity of the illness. The PRISM III score is a valid measure of illness severity in the first

Table 5 Odds ratio, sensitivity, and specificity for admission blood lactate at different concentrations to predict inhospital mortality in critically ill children

\begin{tabular}{|c|c|c|c|c|c|c|c|c|}
\hline Admission blood lactate $\mathrm{mmom} / \mathrm{L}$, & $\mathrm{OR}^{\mathrm{a}}(95 \% \mathrm{Cl})$ & $P$ value & Sensitivity & Specificity & LR+ & LR- & $\mathrm{PV}+$ & PV- \\
\hline$>2.00$ & $4.95(2.27-10.78)$ & $<0.001$ & $94 \%$ & $24 \%$ & 1.2 & 0.25 & 0.55 & 0.80 \\
\hline$>4.00$ & $5.68(3.72-8.68)$ & $<0.001$ & $70 \%$ & $70 \%$ & 2.4 & 0.42 & 0.70 & 0.70 \\
\hline$>6.00$ & $10.02(6.59-15.23)$ & $<0.001$ & $55 \%$ & $89 \%$ & 5.1 & 0.51 & 0.84 & 0.66 \\
\hline$>8.00$ & $16.79(10.37-27.19)$ & $<0.001$ & $43 \%$ & $96 \%$ & 10.1 & 0.60 & 0.91 & 0.63 \\
\hline$>10.00$ & $30.59(16.55-56.53)$ & $<0.001$ & $34 \%$ & $98 \%$ & 19.8 & 0.67 & 0.95 & 0.60 \\
\hline
\end{tabular}

$C l$, confidence interval; $L R+$, likelihood ratio positive; $L R$-, likelihood ratio negative; $O R$, odds ratio; $P V+$, positive predictive value; $P V$-, negative predictive value. ${ }^{a}$ Odds ratios of blood lactate at the levels above the set cut-off points. 
24 hours after admission and reflects the clinical picture of a child during the early admission period [19,23]. Previous studies suggest that PRISM III is an important tool in predicting mortality and clinical outcomes in the pediatric population $[20,24,25]$. The association of admission blood lactate with in-hospital mortality in this study was independent of age, gender, and the severity of illness as assessed by the PRISM III score. The ROC curve analysis in the present study showed that the prognostic accuracy of blood lactate for in-hospital mortality $(\mathrm{AUC}=0.79)$ was similar to that of the PRISM III score (AUC $=0.82$ ). Because blood lactate at admission and the PRISM III score obtained within the first 24 hours after PICU admission are comparable in predicting mortality, we recommend assessing mortality risk with blood lactate at admission because it is simple to use.

Previous studies suggest that there is a confounding relationship between hyperlactatemia and hyperglycemia in nondiabetic critically ill patients [26]. In the present study, there was a significant increase in the blood glucose concentration with an increase in the blood lactate levels, suggesting that hyperglycemia is significantly correlated with hyperlactatemia. We further demonstrated that hyperlactatemia was associated with an increased mortality risk, regardless of the presence of hyperglycemia. This finding suggests that hyperglycemia did not confound the association between the elevated blood lactate level and mortality in this study. In contrast, although hyperglycemia had a significant univariate association with mortality risk, when adjusted for concurrent hyperlactatemia and/or other potential risk factors, hyperglycemia was not a significant predictor of mortality risk in critically ill children.

The accumulation of lactic acid in the blood is generally associated with metabolic acidosis [12,27]. This finding raises the question of whether it is possible that the association of hyperlactatemia with mortality could be at least partially attributed to the occurrence of metabolic acidemia. We demonstrated that hyperlactatemia is significantly associated with in-hospital mortality in critically ill children, even after adjusting for arterial $\mathrm{pH}$ and blood bicarbonate.

This study was designed to determine the optimal relationship between the sensitivity and specificity of blood lactate assessment in predicting mortality and to set appropriate cut-off values for predicting in-hospital mortality. A systematic review conducted on studies of adult patients suggests that all patients with a lactate at admission above $2.5 \mathrm{mmol} / \mathrm{l}$ should be closely monitored for signs of deterioration [1]. In our study, $65.0 \%$ of the children had a lactate concentration $>2.5 \mathrm{mmol} / \mathrm{l}$. Blood lactate displayed a sensitivity of $90 \%$ and a specificity of $38 \%$ in predicting in-hospital mortality at $2.5 \mathrm{mmol} / \mathrm{l}$.
Specificity is one of the main characteristics of a predictive marker. A test with high sensitivity and low specificity carries the risk of many false positives. In the present study, an optimal cut-off for predicting inhospital mortality in critically ill children appears to be a blood lactate level of $5.55 \mathrm{mmol} / \mathrm{l}$, which has a sensitivity of $61 \%$ and a specificity of $86 \%$. Our findings were similar to the results of a previous study conducted in children who were admitted to the PICU with a diagnosis of septic shock, where a lactate value of more than $5 \mathrm{mmol} / \mathrm{l}$ was an accurate predictor of death [28]. Our data, together with previous data, suggest that all critically ill children with high blood lactate at admission should be closely monitored for signs of clinical deterioration and children with low lactate levels should be considered for further monitoring of blood lactate, since serial lactate values may provide better prognostic information $[1,6,9,29]$.

The limitations of the present study include a temporal mismatch in evaluating mortality indicators. We compared the prognostic performance of a lactate value obtained within 2 hours of admission to a PRISM III score, which considers a range of values and includes the worst values obtained in the first 24 hours of admission. The prognostic accuracy of the combination of blood lactate level and PRISM III score was not significantly better than the use of PRISM III alone ( $p=0.135)$, which might be explained by the discrepancy in the timescales for these two methods. Notably, the primary aim of the study was to evaluate the predictive value of blood lactate, when measured as a screening method at admission, to predict mortality in critically ill children. The blood samples for lactate measurements were collected only within the first 2 hours of admission. Thus, serial changes in the lactate levels during the first 24 hours of admission were not evaluated. Trends in lactate concentration over time reflect the clinical response of patients to resuscitation. Serial measurements of lactate levels might improve the sensitivity and specificity of this prognostic test [29]. Further studies are needed to investigate the trends in the changes of lactate values and explore whether the addition of the highest lactate value in the first 24 hours to the PRISM III evaluation improves the prediction of mortality in the critically ill pediatric population.

A second limitation of this study is that the criteria for PICU admission vary widely. Our results might be biased by the fact that we conducted a single-center study. In our study, $77 \%$ of the children had an admission lactate level above $2 \mathrm{mmol} / \mathrm{l}$, and $10.6 \%$ of the children died during the hospital stay. However, only $32 \%$ of children with an admission lactate level between 6 and $10 \mathrm{mmol} / \mathrm{l}$ had been mechanically ventilated. Our data imply that children might be admitted later to the PICU and received less aggressive treatment in our unit. These 
factors may limit the generalizability of our results to health care systems in which children are admitted earlier in their critical illness course and are managed more aggressively. A multicenter study is necessary to confirm our findings.

A third limitation of the study is that during the blood collection period, a substantial number of children received treatment with common PICU therapeutic interventions such as epinephrine. Epinephrine is known to affect lactate levels [30,31]. Unfortunately, we were unable to include these data in our multivariate analyses because we did not have information concerning therapeutic interventions for all children during their transfers to the PICU.

\section{Conclusions}

Our study indicates that the blood lactate levels on admission to the PICU were significantly associated with mortality in critically ill children, even after adjusting for age, gender, and illness severity. A high level of blood lactate upon admission was independently predictive of in-hospital mortality in the pediatric population. These findings extend the knowledge of blood lactate as a clinical biomarker of mortality in critical illness.

\section{Abbreviations}

AUC: Area under the receiver-operating-characteristic curve; $\mathrm{Cl}$ : Confidence interval; MODS: Multi-organ dysfunction syndrome; OR: Odds ratio; PICU: Pediatric intensive care unit; PIM: Pediatric index of mortality; PRISM III: Pediatric risk of mortality III; ROC: Receiver operating characteristic.

\section{Competing interests}

The authors declare that they have no competing interests.

\section{Authors' contributions}

ZJ Bai participated in study design and protocol development. XP Zhu carried out the data analysis and interpretation of data. MX Li and J Pan participated in clinical data collection. J Hua, Y Li, and J Wang participated in the design of the study and coordination. YH Li participated in data analysis, interpretation of data and writing of the manuscript. All authors read and approved the final manuscript.

\section{Acknowledgements}

This work was supported by grants from the Natural Science Foundation for Research Projects in the Colleges and Universities of Jiangsu Province (12KJB320006), the Natural Science Foundation of Jiangsu Province (BK2012604), and the National Natural Science Foundation of China (81370773). Zhenjiang Bai and Xueping Zhu share first authorship.

\section{Author details}

${ }^{1}$ Pediatric Intensive Care Unit, Children's Hospital affiliated to Soochow University, Suzhou, China. ${ }^{2}$ Department of Neonatology, Children's Hospital affiliated to Soochow University, Suzhou, China. ${ }^{3}$ Department of Nephrology, Children's Hospital affiliated to Soochow University, Suzhou, China. ${ }^{4}$ Institute of Pediatric Research, Children's Hospital affiliated to Soochow University, Suzhou, China.

Received: 6 August 2013 Accepted: 21 March 2014

Published: 28 March 2014

\section{References}

1. Kruse O, Grunnet N, Barfod C: Blood lactate as a predictor for in-hospital mortality in patients admitted acutely to hospital: a systematic review. Scand J Trauma Resusc Emerg Med 2011, 19:74.
2. Kang YR, Um SW, Koh WJ, Suh GY, Chung MP, Kim H, Kwon OJ, Jeon K: Initial lactate level and mortality in septic shock patients with hepatic dysfunction. Anaesth Intensive Care 2011, 39:862-867.

3. Nichol AD, Egi M, Pettila V, Bellomo R, French C, Hart G, Davies A, Stachowski E, Reade MC, Bailey M, Cooper DJ: Relative hyperlactatemia and hospital mortality in critically ill patients: a retrospective multicentre study. Crit Care 2010, 14:R25.

4. Martin J, Blobner M, Busch R, Moser N, Kochs E, Luppa PB: Point-of-care testing on admission to the intensive care unit: lactate and glucose independently predict mortality. Clin Chem Lab Med 2013, 51:405-412.

5. Jansen TC, van Bommel J, Bakker J: Blood lactate monitoring in critically ill patients: a systematic health technology assessment. Crit Care Med 2009, 37:2827-2839.

6. Kalyanaraman M, DeCampli WM, Campbell Al, Bhalala U, Harmon TG, Sandiford P, McMahon CK, Shore S, Yeh TS: Serial blood lactate levels as a predictor of mortality in children after cardiopulmonary bypass surgery. Pediatr Crit Care Med 2008, 9:285-288.

7. Juneja D, Singh O, Dang R: Admission hyperlactatemia: causes, incidence, and impact on outcome of patients admitted in a general medical intensive care unit. J Crit Care 2011, 26:316-320.

8. Ramakrishna B, Graham SM, Phiri A, Mankhambo L, Duke T: Lactate as a predictor of mortality in Malawian children with WHO-defined pneumonia. Arch Dis Child 2012, 97:336-342.

9. Hatherill M, McIntyre AG, Wattie M, Murdoch IA: Early hyperlactataemia in critically ill children. Intensive Care Med 2000, 26:314-318.

10. Duke T, Butt W, South M, Karl TR: Early markers of major adverse events in children after cardiac operations. J Thorac Cardiovasc Surg 1997, 114:1042-1052.

11. Siegel LB, Dalton HJ, Hertzog JH, Hopkins RA, Hannan RL, Hauser GJ: Initial postoperative serum lactate levels predict survival in children after open heart surgery. Intensive Care Med 1996, 22:1418-1423.

12. Hatherill M, Waggie Z, Purves L, Reynolds L, Argent A: Mortality and the nature of metabolic acidosis in children with shock. Intensive Care Med 2003, 29:286-291.

13. Duke TD, Butt W, South M: Predictors of mortality and multiple organ failure in children with sepsis. Intensive Care Med 1997, 23:684-692.

14. Morris KP, McShane P, Stickley J, Parslow RC: The relationship between blood lactate concentration, the Paediatric Index of Mortality 2 (PIM2) and mortality in paediatric intensive care. Intensive Care Med 2042-2046, 2012:38.

15. Koliski A, Cat I, Giraldi DJ, Cat ML: Blood lactate concentration as prognostic marker in critically ill children. J Pediatr (Rio J) 2005, 81:287-292.

16. Cheifetz IM, Kern FH, Schulman SR, Greeley WJ, Ungerleider RM, Meliones $J \mathrm{~N}$ : Serum lactates correlate with mortality after operations for complex congenital heart disease. Ann Thorac Surg 1997, 64:735-738.

17. American Academy of Pediatrics, Committee on Hospital Care and Section on Critical Care and Society of Critical Care Medicine, Pediatric Section Admission Criteria Task Force: Guidelines for developing admission and discharge policies for the pediatric intensive care unit. Pediatrics 1999, 103:840-842.

18. Goldstein B, Giroir B, Randolph A: International pediatric sepsis consensus conference: definitions for sepsis and organ dysfunction in pediatrics. Pediatr Crit Care Med 2005, 6:2-8.

19. Pollack MM, Patel KM, Ruttimann UE: PRISM III: an updated pediatric risk of mortality score. Crit Care Med 1996, 24:743-752.

20. Tan GH, Tan TH, Goh DY, Yap HK: Risk factors for predicting mortality in a paediatric intensive care unit. Ann Acad Med Singapore 1998, 27:813-818.

21. Mikkelsen ME, Miltiades AN, Gaieski DF, Goyal M, Fuchs BD, Shah CV, Bellamy SL, Christie JD: Serum lactate is associated with mortality in severe sepsis independent of organ failure and shock. Crit Care Med 2009, 37:1670-1677

22. Gustavsson L, Andersson LM, Brink M, Lindh M, Westin J: Venous lactate levels can be used to identify patients with poor outcome following community-onset norovirus enteritis. Scand J Infect Dis 2012, 44:782-787.

23. Wang JN, Wu JM, Chen YJ: Validity of the updated pediatric risk of mortality score (PRISM III) in predicting the probability of mortality in a pediatric intensive care unit. Acta Paediatr Taiwan 2001, 42:333-337.

24. Brady AR, Harrison D, Black S, Jones S, Rowan K, Pearson G, Ratcliffe J, Parry GJ: Assessment and optimization of mortality prediction tools for admissions to pediatric intensive care in the United kingdom. Pediatrics 2006, 117:e733-e742. 
25. Gemke RJ, van Vught J: Scoring systems in pediatric intensive care: PRISM III versus PIM. Intensive Care Med 2002, 28:204-207.

26. Green JP, Berger T, Garg N, Horeczko T, Suarez A, Radeos MS, Hagar Y, Panacek EA: Hyperlactatemia affects the association of hyperglycemia with mortality in nondiabetic adults with sepsis. Acad Emerg Med 2012, 19:1268-1275.

27. Gunnerson KJ, Saul M, He S, Kellum JA: Lactate versus non-lactate metabolic acidosis: a retrospective outcome evaluation of critically ill patients. Crit Care 2006, 10:R22.

28. Jat KR, Jhamb U, Gupta VK: Serum lactate levels as the predictor of outcome in pediatric septic shock. Indian J Crit Care Med 2011, 15:102-107.

29. Charpie JR, Dekeon MK, Goldberg CS, Mosca RS, Bove EL, Kulik TJ: Serial blood lactate measurements predict early outcome after neonatal repair or palliation for complex congenital heart disease. J Thorac Cardiovasc Surg 2000, 120:73-80.

30. Day NP, Phu NH, Bethell DP, Mai NT, Chau TT, Hien TT, White NJ: The effects of dopamine and adrenaline infusions on acid-base balance and systemic haemodynamics in severe infection. Lancet 1996, 348:219-223.

31. Levy B: Bench-to-bedside review: is there a place for epinephrine in septic shock? Crit Care 2005, 9:561-565.

doi:10.1186/1471-2431-14-83

Cite this article as: Bai et al.: Effectiveness of predicting in-hospital mortality in critically ill children by assessing blood lactate levels at admission. BMC Pediatrics 2014 14:83.

\section{Submit your next manuscript to BioMed Central and take full advantage of:}

- Convenient online submission

- Thorough peer review

- No space constraints or color figure charges

- Immediate publication on acceptance

- Inclusion in PubMed, CAS, Scopus and Google Scholar

- Research which is freely available for redistribution 